\title{
Retour sur la Mission d'information parlementaire française sur les événements de Srebrenica (2001)
}

\section{Pierre Brana}

\section{(2) OpenEdition \\ 1 Journals}

Édition électronique

URL : http://journals.openedition.org/conflits/2203

DOI : 10.4000/conflits.2203

ISSN : $1777-5345$

Éditeur :

CCLS - Centre d'études sur les conflits lilberté et sécurité, L'Harmattan

Édition imprimée

Date de publication : 1 mai 2007

Pagination : 51-62

ISBN : 978-2-296-03084-8

ISSN : 1157-996X

Référence électronique

Pierre Brana, «Retour sur la Mission d'information parlementaire française sur les événements de Srebrenica (2001) », Cultures \& Conflits [En ligne], 65 | printemps 2007, mis en ligne le 04 janvier 2010, consulté le 30 mars 2021. URL : http://journals.openedition.org/conflits/2203 ; DOI : https://doi.org/ $10.4000 /$ conflits.2203 


\section{Retour sur la Mission d'information parlementaire française sur les événements de Srebrenica (2001)}

\section{Pierre BRANA}

Pierre Brana est un ancien député (groupe des députés socialistes et apparentés), membre de la Mission d'information sur les événements de Srebrenica, rapporteur de la Mission d'information sur le Rwanda, membre puis secrétaire de la commission des Affaires étrangères de 1988 à 1993 et de 1997 à 2002 (rapporteur des crédits de la coopération puis du budget du ministère des Affaires étrangères).

$M_{\text {Mission d'information sur les événements de Srebrenica, à la lumière des }}^{\text {on intervention voudrait rendre compte de que fut le traval de la }}$ logiques et des spécificités d'un tel dispositif parlementaire. Le questionnement concerne donc la façon dont des instances publiques - ici le Parlement peuvent contribuer à une meilleure connaissance de la vérité sur un événement de la plus grande importance et de la plus grande gravité. Il concerne également les formes que peut prendre le contrôle du pouvoir exécutif par le Parlement, notamment en matière de relations internationales et de politique de défense, domaines sensibles, s'il en est, dans l'équilibre traditionnel entre l'exécutif et le législatif.

Pour comprendre ce qui va suivre, il est fondamental de commencer par rappeler ce que l'on entend par «commission d'enquête » et « mission d'information ». L'article $140 \mathrm{du}$ règlement de l'Assemblée nationale précise que :

« la création d'une commission d'enquête par l'Assemblée résulte du vote d'une proposition de résolution déposée, renvoyée à la commission permanente compétente, examinée et discutée dans les conditions fixées par le présent règlement. Cette proposition doit déterminer avec précision, soit les faits qui donnent lieu à enquête, soit les services publics ou les entreprises nationales dont la commission doit examiner la gestion». 
« les commissions permanentes assurent l'information de l'Assemblée pour lui permettre d'exercer son contrôle sur la politique du gouvernement. A cette fin, elles peuvent confier à un ou plusieurs de leurs membres une mission d'information temporaire, portant notamment sur les conditions d'application d'une législation. Ces missions d'information peuvent être communes à plusieurs commissions ».

Le règlement indique peu après que la commission permanente demandeuse "doit déterminer avec précision l'objet de la mission pour l'exercice de laquelle le bénéfice des prérogatives attribuées aux commissions d'enquête est demandée».

Dans le cas d'une commission d'enquête comme dans celui d'une mission d'information, si le Garde des sceaux fait savoir que des poursuites judiciaires sont en cours sur les faits ayant motivé le dépôt de la proposition de création d'une commission ou d'une mission, celle-ci ne peut être débattue. Si la discussion a déjà commencé, elle est immédiatement interrompue. De même, si une information judiciaire est ouverte après la création d'une commission ou d'une mission, celle-ci met immédiatement fin à ses travaux.

Les différences entre commission d'enquête et mission d'information apparaissent comme réduites dans les textes, mais il n'en va pas de même dans la pratique. Une commission d'enquête implique une présomption de faute, ou du moins un doute à ce sujet, alors qu'une mission d'information signifie seulement que les députés souhaitent être mieux informés. Comme dans le langage courant, « enquête » et « information » n'ont pas le même sens à l'Assemblée nationale, et le choix entre une commission d'enquête et une mission d'information n'est jamais innocent. Cela est d'autant plus vrai que les personnes recevant une convocation pour une commission d'enquête sont obligées d'y déférer et de prêter serment, ce qui n'est pas le cas devant une mission d'information. La différence est importante, ne serait-ce que sur le plan symbolique.

Mais je voudrais particulièrement attirer l'attention sur le fait que, jusqu'en 1997, d'importantes commissions d'enquête et missions d'information avaient été créées dans presque tous les grands domaines, sauf dans ceux des Affaires étrangères et de la Défense nationale, considérés comme relevant du « domaine réservé » du président de la République. Dès mon retour à l'Assemblée nationale en juin 1997, après une interruption de quatre ans, je retrouvai mon siège à la commission des Affaires étrangères où $j$ 'exerçais la fonction de rapporteur des crédits de la coopération et, par ailleurs, de président du groupe d'amitié parlementaire France - Bosnie-Herzégovine. Je cherchai alors à faire le point sur ce qui s'était réellement passé au Rwanda et dans l'ex-Yougoslavie. Je pris des premiers contacts au sein de mon groupe - le groupe des députés socialistes 
et apparentés - et demandai la création de commissions d'enquête. Il me fut d'abord répondu que cela ne se faisait pas dans le domaine des Affaires étrangères et de la Défense. Ayant cherché le document sur lequel reposait cet interdit et ne l'ayant pas trouvé (et pour cause...), j'en informai mes collègues et relançai la discussion. Finalement, le 3 mars 1998, la commission de la Défense nationale et des forces armées - présidée par Paul Quilès - décida la création d'une Mission d'information sur les opérations militaires menées au Rwanda entre 1990 et 1994 par la France, d'autres pays et l'ONU.

Pour les raisons déjà évoquées, j'aurais préféré la création d'une commission d'enquête, mais la commission de la Défense en ayant décidé autrement, la commission des Affaires étrangères dut se conformer à cette décision. Le 11 mars 1998, elle s'associa donc à cette Mission d'information constituée par les deux commissions, chacune d'elles désignant vingt de ses membres (dix titulaires et dix suppléants) représentant tous les groupes parlementaires de l'Assemblée. Deux rapporteurs furent élus : Bernard Cazeneuve pour la commission de la Défense et moi-même pour celle des Affaires étrangères. L'intitulé officiel de la Mission était : «Enquête sur la tragédie rwandaise (1990-1994)».

Je me suis un peu attardé sur la création de cette Mission d'information parlementaire car il s'agit d'un précédent historique. Jamais, depuis la création de la Ve République, les députés n'avaient décidé de contrôler ainsi l'exécutif dans les domaines sensibles de la Défense et des Affaires étrangères. Jamais, depuis 1958, ils n'avaient osé s'intéresser au domaine dit « réservé » du président de la République. Vu le poids des usages et des rites dans la vie parlementaire, cette décision était d'une grande importance. Il a été dit par la suite que la création de cette Mission avait été la conséquence d'une série d'articles parus dans un grand journal sur la tragédie rwandaise. Pourtant, depuis 1958, il y a eu de nombreuses autres campagnes de presse sur des problèmes de défense ou de politique étrangère, celles-ci n'avaient pas conduit à la création d'une mission parlementaire. Il a aussi été dit que la montagne avait accouché d'une souris, que les résultats n'étaient pas à la hauteur des attentes, que les parlementaires n'étaient pas allés assez loin. Cela est peut-être vrai mais, encore une fois, il s'agissait d'une première, un tabou était brisé et d'autres grandes missions d'information allaient suivre, dont celle sur Srebrenica. Des faits dont la gravité suscitait la mobilisation de nombreux citoyens et engageait directement l'image de la France à l'étranger échappaient enfin, pour partie, au secret d'Etat, les représentants légitimes de la nation pouvant s'en emparer et les étudier. L'exécutif fut obligé de s'expliquer, des documents «secret défense » furent déclassifiés, et une documentation considérable fut rendue publique, ce qui constitue une aubaine pour les chercheurs. Mais, bien entendu, un tel exercice a ses limites. Une mission d'information, comme une commission d'enquête d'ailleurs, a ses règles de fonctionnement et ses contraintes. Il faut savoir que ses pouvoirs d'investigation sont bien inférieurs à ceux d'un simple juge d'instruction. Par exemple, une telle mission n'a pas 
de pouvoir de perquisition et ne peut donc pas vérifier que les documents qui lui ont été fournis sont les plus pertinents.

La création de la Mission d'information parlementaire française sur Srebrenica fut décidée en décembre 2000 par l'Assemblée nationale. Egalement commune aux commissions de la Défense et des Affaires étrangères, elle comportait seulement dix députés (cinq de chaque commission), représentant tous les groupes parlementaires de l'Assemblée nationale. J'aurais, là encore, préféré une commission d'enquête, pour les raisons précédemment indiquées. Je persiste à penser qu'une prestation de serment, par son côté solennel, peut amener certains à faire davantage d'efforts pour exprimer la vérité au plus près. Car, hélas, lors des auditions sur la tragédie du Rwanda et sur celle de Srebrenica, tout ne fut certainement pas dit aux députés et il y eut probablement dans plusieurs dépositions de larges accommodements avec la vérité : l'absence de serment induit, dans les faits, des attitudes différentes chez certaines personnes auditionnées, qui ne confèrent pas le même statut à leur propre parole, elles se sentent donc probablement moins obligées de dire tout ce qu'elles savent. Il est même possible que certaines réponses, étrangement compatibles ou similaires entre elles, aient fait l'objet d'une concertation préalable entre certaines personnes auditionnées. Les partisans de la création d'une simple mission d'information arguent du fait que, les étrangers n'étant pas légalement obligés de venir témoigner devant une commission d'enquête française, ils pourraient être rebutés par l'obligation de prêter serment et ne viendraient donc pas. On se priverait ainsi d'importants témoignages. C'est un argument qui a sa pertinence et qui a d'ailleurs emporté l'adhésion de la majorité, mais à l'égard duquel je reste sceptique : le fait que nous nous contentions d'une mission d'information n'a pas empêché de nombreux étrangers de refuser de venir témoigner, parmi lesquels certains personnages clefs. C'est une des raisons majeures pour lesquelles je continue à préférer la création d'une commission d'enquête pour des tragédies comme celles du Rwanda et de Srebrenica.

Venons-en maintenant à la Mission sur Srebrenica. Je ne reprendrai pas le rapport - ce qui serait long et fastidieux - d'autant qu'il est facilement accessible sur Internet ${ }^{1}$. Je me contenterai d'aborder quelques points qui me paraissent importants. Le premier concerne la mission elle-même. Nous étions dix députés, soit moitié moins que pour le Rwanda, ce qui représente un progrès. En effet, lors des auditions, un trop grand nombre d'intervenants empêche souvent celui qui pose des questions de poursuivre son raisonnement et son objectif. Un débat trop haché est gênant pour pousser dans ses retranchements un acteur ou un témoin réticent à parler. Or, la composition de la Mission, qui reflète fidèlement celle de l'Assemblée nationale, explique à la fois le nombre de membres de la commission et le fait qu'il y ait rarement entente 
sur les questions à poser, chacun réagissant selon sa sensibilité politique. Mais cette diversité, si elle rend plus complexe le travail de la Mission, permet également d'écarter les risques de connivence ou de soumission à une raison d'Etat.

Le second point concerne le choix des auditionnés, qui s'est fait en réunion de la Mission sur proposition des uns et des autres, après débat et vote. Il faut souligner à ce propos qu'une mission ne dure que quelques mois - neuf mois dans le cas de Srebrenica - et que, pendant cette période, les députés ne sont pas libérés de leurs autres obligations : participation aux commissions et séances plénières, présence dans les circonscriptions. Il a donc fallu limiter le nombre d'auditions, ce qui a amené à privilégier les acteurs et les témoins directs au détriment des spécialistes de l'ex-Yougoslavie et des Balkans. Le choix s'est aussi fait de manière relativement empirique, par addition des propositions des uns et des autres plus qu'à l'issue d'une réflexion collective ou en fonction d'une procédure précise. J'ai, pour ma part, établi une liste de propositions sur la base des contacts que j'avais eus en tant que responsable du groupe d'amitié parlementaire France-Bosnie, mais j'ignore comment mes collègues sont parvenus à leurs propres propositions. C'est ce qui peut rendre discutable le choix des personnes auditionnées, ou donner au lecteur l'impression que certaines auditions étaient d'un intérêt limité, redondantes ou privilégiaient les faits, voire l'anecdotique, au détriment des analyses. Il reste que la publication de presque toutes les auditions en annexe du rapport permet de rendre visible le processus de sélection qui conduit de ces procès-verbaux aux conclusions de la Mission d'information et met donc au jour les faiblesses mêmes de notre procédure, tenant à la composition, aux équilibres internes de notre Mission d'information et à la gestion de notre calendrier.

Toutes les personnalités que nous voulions entendre n'ont pu l'être. Le général Rupert Smith, à l'époque commandant la FORPRONU, Madame Sadaka Ogata, responsable du Haut Comité aux réfugiés, ont refusé de venir témoigner. La Mission, dans ce cas, est impuissante, n'ayant aucun moyen de contrainte. Ce refus est d'autant plus regrettable que l'on s'interroge encore aujourd'hui sur les raisons de l'absence du général Rupert Smith à un moment clé de la tragédie, ainsi d'ailleurs que de celle de l'officier de liaison avec l'OTAN détaché auprès du général Janvier, commandant des forces de paix des Nations unies en exYougoslavie. Si on peut comprendre que, le $1^{\text {er }}$ juillet 1995, le général Rupert Smith parte en permission, il est plus surprenant que le commandant de la FORPRONU ne se manifeste à aucun moment de la crise. Nous aurions aimé poser cette question directement à l'intéressé et lui demander également quelle avait été la teneur de ses conversations avec le général Mladic, qu'il a rencontré à deux reprises, le 15 et le 19 juillet 1995. Nous aurions aussi aimé interroger Madame Sadaka Ogata sur l'étonnante inertie des dirigeants du HCR.

Une délégation de la Mission s'est rendue sur le terrain et a procédé à d'autres auditions. Nous n'étions que cinq et nous avons donc pu approfondir 
davantage certaines questions. Nous avons également pu rencontrer des personnes qu'il aurait été difficile de faire venir en France, comme les représentantes de l'association « Femmes de Srebrenica » ou de simples témoins de la situation à Srebrenica qui, dans des discussions informelles, apportaient un éclairage nouveau sur l'état d'esprit et l'atmosphère ambiante. J'avoue attacher une grande importance à ces visites, à ces rencontres sur le terrain. Elles sont souvent essentielles pour comprendre l'enchaînement des événements. Quand nous nous sommes rendus à Srebrenica pour découvrir la topographie des lieux, en empruntant la route du Sud par laquelle les troupes serbes sont arrivées, il est apparu qu'étant donné l'étroitesse de la route située dans un défilé encaissé, une cinquantaine d'hommes entrânés, déterminés et bien armés, avec deux ou trois blindés, auraient pu bloquer l'avancée des soldats serbes. Cette constatation a pesé lourd dans le rapport, notamment pour déterminer les lacunes militaires du bataillon néerlandais mais aussi l'énorme responsabilité prise en refusant aux Bosniaques le droit de se battre et en démantelant leur faible dispositif défensif (remise d'armes lourdes : tanks, pièces d'artillerie, etc.)

Aux auditions et aux visites sur le terrain s'ajoute la recherche de documents. Comme dans le cas du Rwanda, tous les documents que nous avons eus en notre possession ont été publiés en annexe du rapport : des notes des ministères de la Défense et des Affaires étrangères jusqu'aux rapports de l'ONU, en passant par les déclarations et les communiqués de l'époque. Cela représente une documentation importante qui sera certainement utile aux chercheurs. Mais elle est incomplète. A l'évidence, tout ne nous a pas été remis. Si nous n'avons pas essuyé de refus sur la demande précise de telle ou telle note, on peut raisonnablement estimer que, parmi les documents qui nous ont été fournis, certains peu utiles à nos travaux ont été substitués à d'autres beaucoup plus essentiels. C'est ce que le rapport évoque, dans le style policé habituel à ce genre de travail, en regrettant que le ministère de la Défense ait « suivi une ligne plutôt floue dans sa politique de communication des documents », et en ajoutant :

«si la Mission d'information se réjouit d'avoir obtenu une partie des notes internes du ministère, qu'elle avait demandées, elle s'étonne tout autant d'avoir reçu des documents sans doute de valeur, mais assez peu utiles à ses travaux. Heureusement, ces manques ont pu être palliés, dans certains cas, par la collaboration de certaines organisations non gouvernementales ».

Par ailleurs, l'ONU ne nous a pas fourni toutes les informations que nous lui avions demandées et l'OTAN a purement et simplement refusé de répondre à nos demandes. Ceci donne un aperçu des difficultés que peut rencontrer une mission d'information parlementaire pour effectuer correctement son travail, et ce, bien qu'elle ait un caractère officiel et jouisse en théorie de la bénédiction des autorités. 
Au sein de la Mission, les débats se sont pour la plupart bien passés. Le fait de travailler ensemble pendant neuf mois, à raison d'une séance hebdomadaire en moyenne, ne pousse pas à des affrontements permanents. Des évolutions se dessinent, on note des rapprochements, des divergences aussi. Au moment de la rédaction du rapport final, des votes interviennent parfois pour trancher entre telle ou telle analyse, voire entre deux phrases ou même deux mots différents. Le plus souvent, un compromis rédactionnel est trouvé. Cette pratique du compromis est une constante de ces missions car nous sommes toujours à la recherche d'un équilibre, d'autant plus nécessaire que c'est par un vote final que le rapport de la mission d'information est adopté pour être transmis à l'Assemblée et aux pouvoirs publics. Le dispositif est collectif, la pratique qui le caractérise l'est tout autant, et le rapport qui en est issu est marqué par cette matrice.

Toutefois, l'examen du cas du commandant de la FORPRONU a entraîné des divergences d'appréciation persistantes. Le texte du rapport est sans ambiguité :

« Nul doute que les atermoiements du commandant de la force de l'ONU, l'erreur manifeste du jugement porté à l'égard de son interlocuteur, le général Mladic, ont joué un rôle dans le drame, de même que le rejet par le général Nicolaï des demandes d'appui aérien antérieures au 9 juillet qui ne parviendront jamais jusqu'à Zagreb. Notamment, le 10 juillet au soir, le général Janvier aurait dû déclencher l'appui aérien rapproché puisqu'étaient réunies toutes les conditions, extrêmement restrictives pourtant, posées par les textes au déclenchement de cette procédure. Les casques bleus avaient été directement attaqués, un ultimatum avait été donné aux Serbes, qui l'ont violé ».

Mais cette conclusion n'a pas obtenu l'accord de deux membres de la mission, et non des moindres, puisqu'il s'agissait de Monsieur François Léotard, co-rapporteur au début de la Mission, avant qu'il ne soit nommé représentant de l'Union européenne en Macédoine, et de son successeur Monsieur René André. Tous les deux ont estimé par lettre que les critiques envers le général Janvier ne prenaient pas en compte une réalité complexe «toujours difficile à mesurer lorsque l'on juge de loin et avec le recul du temps une décision opérationnelle», pour reprendre les termes de Monsieur René André.

Madame Marie-Hélène Aubert et moi-même avons fait remarquer qu'aucun élément ne permettait d'exclure l'hypothèse qu'un accord aurait été conclu entre les généraux Janvier et Mladic sur l'absence de frappes aériennes lors de leur rencontre, le 4 juin 1995, à Zvornik. Nous avons donc déclaré que nous ne pouvions partager la conviction exprimée par les rapporteurs et soutenue par la majorité de la Mission selon laquelle le général Janvier n’avait pas accédé aux demandes du général Mladic, à savoir la libération des otages fran- 
çais détenus par les Serbes contre l'absence de frappes aériennes. Nous demandions simplement que la Mission ait la même attitude, sur ce point, qu'à l'égard de l'hypothèse selon laquelle le gouvernement de Bosnie aurait abandonné Srebrenica en échange des faubourgs serbes de Sarajevo. En effet, la mission a sagement indiqué dans le rapport qu'en l'absence de preuve, elle n'était pas en mesure de trancher cette question. A nos yeux, elle aurait dû, par souci d'équité et d'homogénéité, avoir la même position au sujet de l'hypothèse selon laquelle l'absence de frappes aériennes serait la contrepartie de la libération des otages français un mois auparavant.

Par ailleurs, je regrettai et je regrette toujours personnellement que le terme de "génocide » n'ait pas été explicitement employé dans le rapport à l'encontre de Mladic et Karadzic. Je crois qu'utiliser le mot « génocide » permet de qualifier clairement ce qui s'est passé à Srebrenica. Ne s'agit-il pas d'une tentative visant, selon un plan concerté (la mise au point des moyens de transport nécessitait en effet une logistique affirmée), à éliminer en totalité les hommes parce qu'ils étaient Bosniaques, dont 7000 à 8000 ont été tués ? D’ailleurs, depuis notre Mission, le TPIY a jugé qu'un génocide avait été commis à Srebrenica, la plupart des accusés pour Srebrenica ont été inculpés de génocide et deux d'entre eux ont été condamnés pour complicité de génocide : le général Krstic en appel le 19 avril 2004 et Vidoje Blagojevic en première instance le 17 janvier 2005.

Mais je voudrais revenir sur une question que nous avons rencontrée tout au long des réunions de la Mission, comme cela avait été le cas lors des débats sur la tragédie rwandaise : celle des responsabilités de la France et de l'armée française. Est-ce que reconnaître des erreurs, des fautes même, affaiblit notre pays et porte ombrage à nos armées ? Il s'agit là d'un vieux débat qui remonte à l'affaire Dreyfus mais qui reste d'actualité, je peux en témoigner pour l'avoir constaté tant lors de la mission sur le Rwanda que lors de celle sur Srebrenica. On peut dire que, globalement, deux tendances s'expriment à ce sujet parmi les députés. Certains sont partisans d'une grande modération dès qu'il est question des responsabilités de la France et de son armée. Ces députés minimisent systématiquement les erreurs ou les fautes commises, estimant que les rendre publiques ternit l'image de la France dans le monde. Et quand ils ne peuvent éviter que soit traité tel ou tel fait précis, ils demandent - souvent avec force et insistance - que soit soulignée immédiatement en contrepoint l'importance de l'engagement de notre pays tant en termes d'effectifs que sur le plan humanitaire et de l'action pacificatrice de notre armée.

L'autre tendance - à laquelle je me flatte d'appartenir - pense au contraire que reconnaitre publiquement des dysfonctionnements, des erreurs, des fautes, voire des crimes, non seulement ne diminue pas l'image d'un pays, mais peut au contraire la grandir. Et ce n'est pas un hasard si le président des EtatsUnis ou le Secrétaire général de l'ONU ont présenté regrets et excuses sous 
forme de mea culpa, l'un au sujet du Rwanda, l'autre pour Srebrenica. Mais reconnaître que l'on s'est trompé, ou pire, que l'on a commis une faute, ne semble pas encore faire partie de la culture française. Bien sûr, il ne s'agit pas, a contrario, de tomber dans la repentance ou l'autoflagellation permanente, mais de dire clairement ce qui s'est passé, sans le minimiser ni l'exagérer.

Voilà pourquoi, pour terminer, je voudrais répondre par anticipation à une question qui m'est fréquemment posée : quel regard peut-on porter, avec le recul du temps, sur ces grandes missions d'information ? Je ne reviendrai pas sur ma préférence pour les commissions d'enquête, plus solennelles et, par là même, mieux adaptées selon moi à des questions graves telles que des interventions militaires dans lesquelles la responsabilité de la France est engagée directement ou indirectement. Je ne reviendrai pas non plus sur les limites d'une mission d'information : faibles pouvoirs d'investigation, durée réduite (encore qu'elle puisse dépasser, et c'est l'un de ses rares avantages, six mois imposés aux commissions d'enquête), collaboration plus ou moins active des ministères nationaux et des institutions internationales, manque de suivi par l'exécutif des recommandations ou des enseignements à tirer.

Malgré toutes ces réserves, mon appréciation reste positive. En premier lieu, les députés membres de la mission, au fur et à mesure qu'ils découvrent les tenants et aboutissants d'événements qu'ils ne connaissaient pas mieux que leurs concitoyens, en parlent autour d'eux, et notamment au sein de leurs groupes parlementaires respectifs, et contribuent ainsi à la sensibilisation de l'ensemble des parlementaires et de la classe politique. Ensuite, le rapport final est rendu public. Même s'il est contestable ou incomplet, il a le grand mérite, par le canal des médias, d'être porté à la connaissance du grand public, de susciter réactions, discussions et controverses, ce qui ne peut qu'être bénéfique pour la recherche de la vérité. Etant donné qu'il comporte toujours de copieuses annexes avec des documents déclassifiés ou difficiles à obtenir, ce rapport représente aussi une source de renseignements particulièrement utiles pour les chercheurs, les journalistes d'investigation et même la justice. Enfin, les travaux d'une mission sont suivis avec grande attention par l'exécutif, la présidence de la République et le gouvernement, mais aussi par les diplomates, les militaires, les hauts fonctionnaires et tous ceux qui exercent ou ont exercé des responsabilités dans le domaine examiné. Je peux témoigner que beaucoup s'inquiètent - il n'est jamais agréable de se faire « épingler »-, s'interrogent et même tirent des enseignements pour le présent et l'avenir.

Je crois que s'il y avait davantage de missions d'information parlementaire et surtout de commissions d'enquête, si le Parlement exerçait davantage son pouvoir de contrôle - reconnu par la Constitution - dans les domaines de la Défense nationale et des forces armées comme dans celui des Affaires étrangères, l'exécutif serait plus attentif aux réactions de la représentation nationale et modifierait quelquefois son comportement sur le terrain. Je suis persuadé que 
l'absence d'une pression maintenue explique que les recommandations contenues dans le rapport sur le génocide au Rwanda n'aient pas été suivies par l'exécutif. Je le regrette profondément car leur application aurait représenté une avancée décisive vers plus de démocratie et une protection importante contre tout risque de renouvellement d'une pareille tragédie. Je pense en particulier à la proposition d'améliorer la transparence des décisions concernant la participation à des opérations militaires conduites en dehors du territoire national et à l'amélioration du contrôle du Parlement, tant par une meilleure utilisation des moyens actuels que par la création de nouveaux mécanismes.

Aujourd'hui, la Constitution française ne laisse qu'une place très limitée au Parlement en matière d'emploi des forces militaires. Un vote n'est requis que pour une déclaration de guerre en bonne et due forme, ce qui, avec les conflits de notre époque, est complètement obsolète. Il faudrait donc modifier la Constitution et créer des modalités d'autorisation des opérations militaires extérieures par le Parlement, y compris lorsqu'il s'agit de la participation de nos troupes à une force de l'ONU. Le rapport sur Srebrenica n'avance pas de manière aussi explicite des recommandations concrètes adressées à l'exécutif français et à l'ONU. Des collègues de la Mission m’ont répondu que ces recommandations apparaissent en creux tout au long du rapport. C'est en partie vrai, mais les rappeler et les regrouper dans un seul chapitre, même si cela peut paraître redondant, permet de leur donner plus de force, d'en faire un message explicite et vigoureux adressé au président de la République, au gouvernement et à l'ONU.

On ne cesse de parler de réformes de l'ONU depuis les génocides au Rwanda et à Srebrenica. Il faut que celles-ci permettent à l'ONU d'être plus efficace, de voir ses procédures allégées, de rendre ses représentants sur le terrain plus autonomes. Il faut également espérer que les casques bleus, sous une direction véritablement unique, cessent d'être tiraillés entre plusieurs chaînes de commandement, nationales et onusienne. Il serait ainsi possible qu'à côté du légitime souci de préserver l'intégrité physique des soldats engagés, se fasse jour une tout aussi légitime attention à la protection des civils, comme priorité de l'engagement des forces onusiennes. Pour notre pays, je pense qu'il faudra encore beaucoup de missions d'information et de commissions d'enquêtes pour qu'une évolution se produise. A vrai dire, je crains même plutôt que le processus initié en 1997 ne s'arrête. Il faudrait qu'un plus grand nombre de députés s'intéressent activement aux questions de politique étrangère. Mais c'est un secteur qui mobilise peu l'hémicycle, comme l'opinion publique d'ailleurs. Les parlementaires sont les reflets des électeurs.

Il faut souhaiter le développement d'une culture de l'investigation qui appartient trop peu à nos traditions parlementaires : celle-ci permettrait sans doute de faire participer les parlementaires à la formation de l'opinion publique, et de renforcer ainsi leurs liens avec les citoyens. Or, la culture d'investi- 
gation - dont l'un des effets serait aussi de renforcer l'indépendance du Parlement - ne peut progresser et se développer que par la multiplication des pratiques qui lui sont associées. C'est alors seulement que les missions d'information pourront être suivies d'effets, que les recommandations donneront lieu à une début de mise en ouvre, voire que des suites judiciaires seront envisageables, bref, que l'exécutif se sentira quelque peu engagé par le résultat du travail parlementaire. 